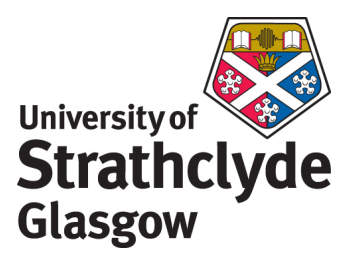

Brown, Blair and McInnes, C.R. and Allouis, E. (2010) Dynamic autonomous intelligent control of an asteroid lander. Proceedings of the Institution of Mechanical Engineers, Part G: Journal of Aerospace Engineering, 224 (8). pp. 865-879. ISSN 0954-4100 (In Press)

http://strathprints.strath.ac.uk/26798/

This is an author produced version of a paper published in Proceedings of the Institution of Mechanical Engineers, Part G: Journal of Aerospace Engineering, 224 (8). pp. 865-879. ISSN 0954-4100. This version has been peer-reviewed but does not include the final publisher proof corrections, published layout or pagination.

Strathprints is designed to allow users to access the research output of the University of Strathclyde. Copyright $(C$ and Moral Rights for the papers on this site are retained by the individual authors and/or other copyright owners. You may not engage in further distribution of the material for any profitmaking activities or any commercial gain. You may freely distribute both the url (http://strathprints.strath.ac.uk) and the content of this paper for research or study, educational, or not-for-profit purposes without prior permission or charge. You may freely distribute the url (http://strathprints.strath.ac.uk) of the Strathprints website.

Any correspondence concerning this service should be sent to The Strathprints Administrator: eprints@cis.strath.ac.uk 


\title{
DYNAMIC AUTONOMOUS INTELLIGENT CONTROL OF AN ASTEROID LANDER
}

\author{
Blair Brown* - Colin McInnes* - Elie Allouis** \\ *Advanced Space Concepts Laboratory \\ Department of Mechanical Engineering \\ University of Strathclyde, Glasgow, UK \\ **EADS Astrium ltd, Stevenage, UK \\ E-mail: blair.brown@strath.ac.uk
}

November 24, 2009

\begin{abstract}
One of the future flagship missions of the European Space Agency (ESA) is the asteroid sample return mission Marco-Polo. Although there have been a number of past missions to asteroids, a sample has never been successfully returned. The return of asteroid regolith to the Earth's surface introduces new technical challenges. This paper develops attitude control algorithms for the descent phase onto an asteroid in micro-gravity conditions and draws a comparison between the algorithms considered. Two studies are also performed regarding the Fault Detection Isolation and Recovery (FDIR) of the control laws considered. The potential of using Direct Adaptive Control (DAC) as a controller for the surface sampling process is also investigated. Use of a DAC controller incorporates increased levels of robustness by allowing realtime variation of control gains. This leads to better response to uncertainties encountered during missions.
\end{abstract}

Keywords: autonomy, asteroid, direct adaptive control, fault detection isolation and recovery

\section{Introduction}

A number of missions have been and are being conducted with the aim of studying Near Earth Objects (NEO) such as asteroids and comets, e.g. Rosetta[1], Deep-Impact[2], and Hayabusa[3]. A key aim is now to return a sample of the constituent material from a NEO. ESA plans to achieve this goal as part of the Marco-Polo mission.

Alongside asteroid and comet observation two of the previous missions also carried landers with the aim of traversing across the asteroid surface. In the case of Hayabusa this was the MINERVA mini-lander[4] intended to hop from one location to the next on a ballistic trajectory using simple DC torquers for attitude control. The second is the Rosetta mission and its lander Philae[5]. 
Philae will take in situ measurments on the surface of the target comet with descent attitude stabilisation performed using an internal reaction wheel with cold jet thrusters available if the descent velocity become too large.

Due to the fact this is a new engineering problem that has never been accomplished before there are new technical challenges to be overcome. The key issues are associated with guidance, navigation, and control (GNC) in micro-gravity necessary for descent and landing. Also, sustained presence on the surface of the NEO for the scientific phase to take place is required.

Other missions that draw parallel with this work are the lander descent GNC for the Apollo lunar and Viking Mars programs. In the case of the Viking lander there had to be fully autonomous descent and landing due to the large distance between the lander and the ground station [6]. Currently, research is being conducted into new forms of GNC for descent optimisation and trajectory following such as light detection and ranging (LIDAR) GNC [7] [8] [9]. ESA has recently built a specialised facility for testing such technologies [10] [11].

The first section of this paper deals with different methods for the attitude control of a NEO asteroid lander based on the Technology Reference Study (TRS) performed by EADS Astrium Ltd for the ESA Marco-Polo mission [12]. A comparison is drawn between different control methods and a discussion is included to better understand the merits of each control algorithm.

The controllers will then be tested for their applicability to fault detection, isolation and recovery (FDIR) under two failure scenarios. Firstly when the mechanical properties of the lander leg changes (e.g. a stuck leg) and secondly when one attitude thruster is assumed to fail completely. Comparison and discussion of the merits of the controllers will be presented. It is shown that there is a trade-off to be made between the controllers with regards fuel and settling time required. In the case of a Direct Adaptive Control (DAC) method it is demonstrated that this is the only controller capable of maintaining an acceptable attitude during the failures.

Finally, a short study investigating the applicability of a DAC algorithm to a hold-down scenario during sampling will be demonstrated and discussed. The paper then demonstrates that DAC is remarkably effective in adaptively stabilising these scenarios in spite of the broad range of unknown disturbances present.

\section{Lander Representation}

This paper focuses on control laws applied to the attitude stabilization of the Marco Polo asteroid lander, adapted from the Astrium near earth asteroid sample return technology reference study (NEA-SR TRS) shown in figure 1. A relatively basic three degree of freedom (3-DOF) representation of the lander has been adopted for modeling purposes. Fig.2 depicts the layout of the lander as it exists in the model, where the y-axis is positive out of the page. Each thruster has a mean thrust value of 10 Newtons, and are the method of attitude correction. The contractible spring constant is 1250 Newton/meter modeling 
the leg resistance to compression only. During the leg reaction to compression, the extension constant is modeled by a ratchet constant, 22500 Newton/meter, in addition to the existing spring. The natural length of each lander leg (before deformation) is 1 meter and the mass of each leg is $7.3 \mathrm{~kg}$. Laterally the length of the lander base is $2.8 \mathrm{~m}$ and the mass of the base only is $985.4 \mathrm{~kg}$. These parameter values denoted in the Fig. 2 are constant and are specified in table 1.

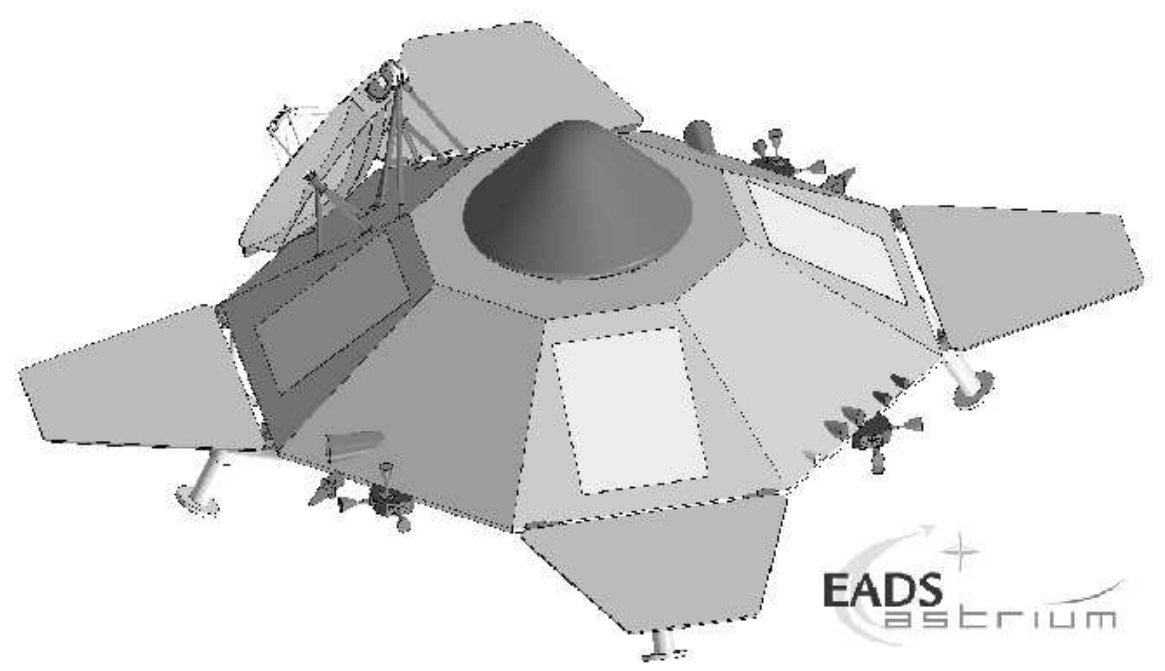

Figure 1: Astrium TRS lander. Courtesy of EADS Astrium ltd

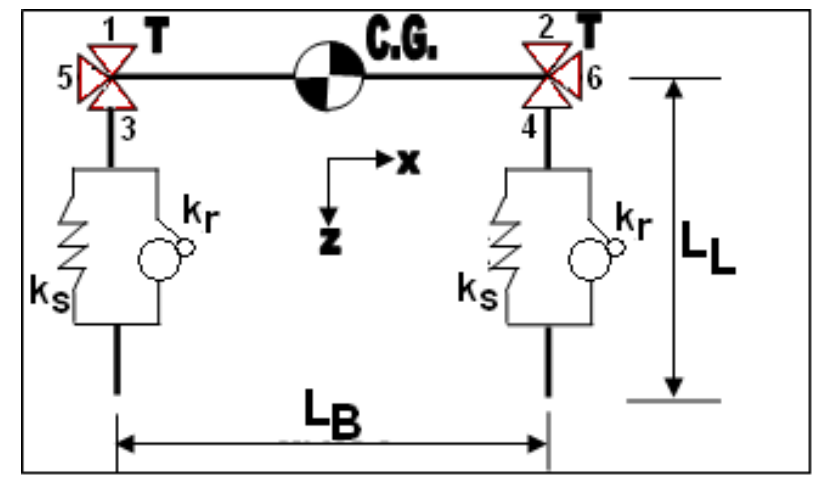

Figure 2: Model lander schematic

Several assumptions have been made about the lander:

- The mass of the lander legs $\left(m_{L}\right)$ is much less than the mass of the lander base $\left(m_{B}\right)$. 
Table 1: Lander properties

\begin{tabular}{|c|c|c|}
\hline Parameter & Value & Unit \\
\hline Thrust $(\mathrm{T})$ & 10 & $\mathrm{~N}$ \\
\hline Spring Const $\left(k_{s}\right)$ & 1250 & $\mathrm{~N} / \mathrm{m}$ \\
\hline Ratchet Const $\left(k_{r}\right)$ & 22500 & $\mathrm{~N} / \mathrm{m}$ \\
\hline Leg Natural Length $\left(L_{L}\right)$ & 1 & $\mathrm{~m}$ \\
\hline Leg Stroke Length $\left(L_{s}\right)$ & 0.3 & $\mathrm{~m}$ \\
\hline Base Length $\left(L_{B}\right)$ & 2.8 & $\mathrm{~m}$ \\
\hline Base Mass $\left(m_{B}\right)$ & 985.4 & $\mathrm{~kg}$ \\
\hline Leg Mass $\left(m_{L}\right)$ & 7.3 & $\mathrm{~kg}$ \\
\hline
\end{tabular}

- The center of gravity (C.G.) of the lander lies at the mid lateral point of the lander base.

- The lander base is rigid and does not deform.

- The lander legs are at 90 degrees to the base.

- The lander legs deform only in one axis, the z-axis in body axes.

The thruster layout associated with the lander is arranged in order to allocate thrusters to specific axes. The thrusters are numbered 1-6 in Fig.2 where; 1 and 3 individually or together produce a moment in a positive sense around the yaxis, 2 and 4 produce a moment in the negative sense, and 5 and 6 individually produce a translation positively and negatively respectively in the $\mathrm{x}$-axis.

\section{Computational Model}

The lander simulation consists of a number of ordinary differential equation (ODE) solvers running at specific time intervals to integrate the relevant equations of motion in two dimensions using three degrees of freedom. Discontinuities in the dynamics of the system, such as leg impacts, are defined heuristically and captured using Matlab algorithms. All the equations of motion are defined in body axes, where the axis system is defined in Fig.3. The dynamics of the model allow the system to exist in three different states, requiring three different sets of equations of motion and three separate ODE solvers. The first of these states is free-flight. This is the state the lander will assume in descent or rebound where neither leg has contact with the asteroid. This state has 14 parameters to be solved for and these are specified in equation (1). 


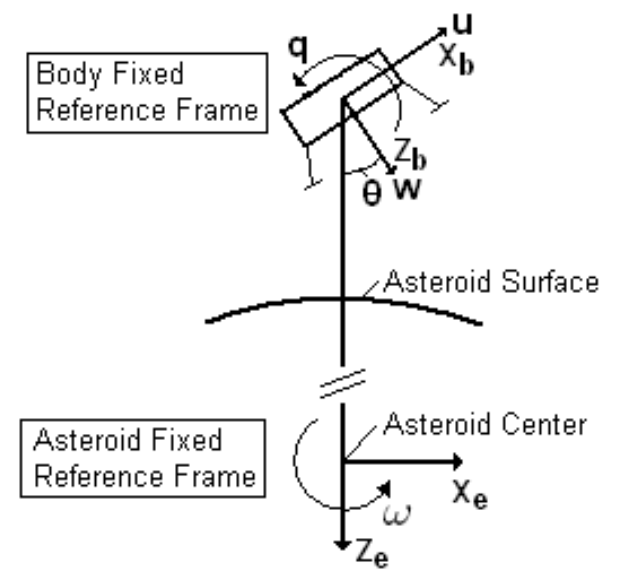

Figure 3: Model axes definitions

$$
\begin{aligned}
\ddot{x}_{\text {base }} & =\frac{F x_{\text {base }}}{m_{B}}-q w_{\text {base }}-g \sin (\theta) \\
\ddot{z}_{\text {base }} & =\frac{F z_{\text {base }}}{m_{B}}+q u_{\text {base }}+g \cos (\theta) \\
\ddot{x}_{\text {leg }}^{L} & =\frac{F x_{\text {leg }}}{m_{E}}-q w_{\text {leg }}^{L}-g \sin (\theta) \\
\ddot{z}_{\text {leg }}^{L} & =\frac{F z_{\text {leg }}}{m_{\text {I }}}+q u_{\text {leg }}^{L}+g \cos (\theta) \\
\ddot{x}_{\text {leg }}^{R} & =\frac{F x_{\text {leg }}}{m_{K}}-q w_{\text {leg }}^{R}-g \sin (\theta) \\
\ddot{z}_{\text {leg }}^{R} & =\frac{F z_{\text {leg }}}{m_{I}}+q u_{\text {leg }}^{R}+g \cos (\theta) \\
\ddot{\theta} & =\frac{M}{I}
\end{aligned}
$$

where $q$ is the angular rate of the lander, $u$ and $w$ are the components of velocity in the x-axis and z-axis respectively, and $\theta$ is the attitude error. $M$ is the resultant moment acting on the body and $I$ is the moment of inertia of the body.

The leg forces are defined such that the force differs depending on the sense of the leg contraction or extension as described below:

$$
F z_{\text {leg }}^{L}=\left\{\begin{array}{c}
k_{s} \cdot\left(\left(z_{\text {base }}-z_{\text {base }}^{\text {rest }}\right)-\left(z_{\text {leg }}^{L}-z_{\text {leg }}^{L r e s t}\right)\right) \\
\dot{z}_{\text {leg }}^{L}>0 \\
k_{s} \cdot\left(\left(z_{\text {base }}-z_{\text {base }}^{\text {rest }}\right)-\left(z_{\text {leg }}^{L}-z_{\text {leg }}^{\text {Lrest }}\right)\right) \\
+k_{r} \cdot\left(\left(z_{\text {base }}-z_{\text {bast }}^{\text {rest }}\right)-\left(z_{\text {leg }}^{\text {Leg }}-z_{\text {leg }}^{\text {Lrest }}\right)\right. \\
\left.-\left(L_{L}-L_{S}\right)\right) \\
\dot{z}_{\text {leg }}^{L} \leq 0
\end{array}\right.
$$




$$
F z_{\text {leg }}^{R}=\left\{\begin{array}{c}
k_{s} \cdot\left(\left(z_{\text {base }}-z_{\text {base }}^{\text {rest }}\right)-\left(z_{\text {leg }}^{R}-z_{\text {leg }}^{\text {Rrest }}\right)\right), \\
\dot{z}_{\text {leg }}^{R}>0 \\
k_{s} \cdot\left(\left(z_{\text {base }}-z_{\text {base }}^{\text {rest }}\right)-\left(z_{\text {leg }}^{R}-z_{\text {leg }}^{\text {Rrest }}\right)\right)+ \\
k_{r} \cdot\left(\left(z_{\text {base }}-z_{\text {base }}^{\text {rest }}\right)-\left(z_{\text {leg }}^{R}-z_{\text {leg }}^{\text {Rrest }}\right)\right) \\
\left.-\left(L_{L}-L_{S}\right)\right), \\
\dot{z}_{\text {leg }}^{R} \leq 0
\end{array}\right.
$$

where all the terms are in body axes. Terms defining a difference in the $z$ locations of the base $\left(z_{\text {base }}\right)$ and legs $\left(z_{\text {leg }}^{L}\right.$ and $\left.z_{\text {leg }}^{R}\right)$ from the base $\left(z_{\text {base }}^{\text {rest }}\right)$ and leg $\left(z_{\text {leg }}^{\text {Lrest }}\right.$ and $\left.z_{\text {leg }}^{\text {Rrest }}\right)$ rest positions are multiplied by either the spring constant only in compression, or the spring and ratchet constant in extension to produce the leg forces that act on the system. During the extension phases of the simulation (i.e. $\dot{z}_{l e g} \leq 0$ ) it is necessary to include a term that simulates the maximum deformation of the spring/ratchet system $\left(L_{L}-L_{S}\right.$, as defined in table 1) so the extension forces only act when appropriate. The reason the ratchet force acts solely in one direction is to reduce the leg extension and absorb the kinetic energy from the decent of the lander when contact with the asteroid surface is made.

The component of force in the z-axis, in body axes $\left(F z_{\text {base }}\right)$, acting on the lander base is the sum of the components of forces currently in the left $\left(F z_{\text {leg }}^{L}\right)$ and right $\left(F z_{\text {leg }}^{R}\right)$ legs in addition to the thruster forces $(\mathrm{T})$ :

$$
F z_{\text {base }}=F z_{\text {leg }}^{L}+F z_{\text {leg }}^{R}+T
$$

The component of force in the $\mathrm{x}$-axis, in body axes, acting on the lander base is equal to the component of force in the $\mathrm{x}$-axis, in body axes, acting on the lander legs and is given by:

$$
F x_{\text {base }}=F x_{\text {leg }}^{L}+F x_{\text {leg }}^{R}+T
$$

where $F x_{\text {leg }}^{L}=F x_{\text {leg }}^{R}=0$ since the legs are perpendicular to the base.

The second dynamic state is when there is only one leg in contact with the asteroid. This can be in a descent scenario or in the rebound ascent phase. In this system there are 10 states to be solved for and can be interpreted as equation (1) minus two of the equations corresponding to one of the legs, which is in contact with the asteroid surface. In this case the resultant force on the base $F z_{\text {base }}$ is defined differently as:

$$
F z_{\text {base }}=\left\{\begin{array}{c}
\left(-k_{s} \cdot\left(z_{\text {base }}-z_{\text {base }}^{\text {rest }}\right)\right)+F z_{\text {leg }}^{\text {free }} \\
\dot{z}_{\text {base }}>0 \\
\left(-k_{s} \cdot\left(z_{\text {base }}-z_{\text {base }}^{\text {rest }}\right)\right) \\
+\left(-k_{r} \cdot\left(z_{\text {base }}-z_{\text {base }}^{\text {rest }}\right)\right)+F z_{\text {leg }}^{\text {free }} \\
\dot{z}_{\text {base }} \leq 0
\end{array}\right.
$$

and the force of the free leg: 


$$
F z_{\text {leg }}^{\text {free }}=\left\{\begin{array}{c}
k_{s} \cdot\left(\left(z_{\text {base }}-z_{\text {base }}^{\text {rest }}\right)-\left(z_{\text {leg }}^{\text {free }}-z_{\text {leg }}^{\text {FreeRest }}\right)\right), \\
\dot{z}_{\text {leg }}^{\text {free }}>0 \\
k_{s} \cdot\left(\left(z_{\text {base }}-z_{\text {base }}^{\text {rest }}\right)-\left(z_{\text {leg }}^{\text {free }}-z_{\text {leg }}^{\text {FreeRest }}\right)\right) \\
+\left(-k_{r} .\left(\left(z_{\text {base }}-z_{\text {bases }}^{\text {res }}\right)-\left(z_{\text {leg }}^{\text {free }}-z_{\text {leg }}^{\text {FreeRest }}\right)\right)\right) \\
\left.-\left(L_{L}-L_{S}\right)\right), \\
\dot{z}_{\text {leg }}^{\text {ree }} \leq 0
\end{array}\right.
$$

where the same analysis applies as before regarding the leg compression and expansion in the case of the application of the ratchet. Here:

$$
F x_{\text {base }}=F x_{\text {leg }}^{\text {free }}+T
$$

where $F x_{\text {leg }}^{\text {free }}=0$ due to the legs being perpendicular to the base.

The third system state is when both lander legs are in contact with the asteroid surface. In this system state there are 6 parameters to be solved for, these are specified in equation (2).

$$
\begin{aligned}
\ddot{x}_{\text {base }} & =\frac{F x_{\text {base }}}{m_{\text {base }}}-q w_{\text {base }}-g \sin (\theta) \\
\ddot{z}_{\text {base }} & =\frac{F z_{\text {base }}}{m_{\text {base }}}+q u_{\text {base }}+g \cos (\theta) \\
\ddot{\theta} & =\frac{M}{I}
\end{aligned}
$$

where the forces obey the usual conventions for application of the ratchet force.

In all three cases $M$, the moment applied around the center of gravity of the lander is defined by the difference in the forces in the legs and the distance between them in addition to the moment produced by the control thrusters.

$$
M=\left(F z_{\text {leg }}^{L}-F z_{\text {leg }}^{R}\right) \cdot L_{B}+M_{T}
$$

where $M_{T}$ is the moment produced by the thrusters.

The points at which the model switches between the set of dynamic equations are defined heuristically. The descent or rebound scenarios terminate when either of the $\mathrm{z}$ components of the legs in inertial axes equals the radius of the asteroid. The descent phase in which one leg has contact finishes when the $\mathrm{z}$ component of the other leg in inertial axes equals the radius of the asteroid. The two leg contact phase ends when either; the rebound force in both legs is great enough to overcome the weight of the entire lander, inducing a rebound, or the lander finishes its transient response and comes to rest on the asteroid surface. The initial rebound (one leg still in contact with the asteroid) phase ends when the force acting on the leg still in contact with the asteroid is greater than the weight of one leg. In any simulation if the attitude of the lander $(\theta)$, is small $(\leq 0.5$ degrees) then the one leg descent and rebound phases are neglected and the model moves onto the next two leg phase. 
The model continues in an iterative loop until the lander is at rest on the surface of the asteroid or alternatively falls over on its side due to instability. These processes are demonstrated in Fig.4; in Fig.4(a) a descent and one rebound can be seen starting from an initially vertical attitude, in Fig.4(b) the effect of adding a one degree attitude error to the descent and rebound causes a subsequent tumbling of the lander. In both cases there is no attitude control. In Fig. 4 the solid bold line indicates the center of mass of the base and the two dashed lines indicate the feet positions during the simulation.
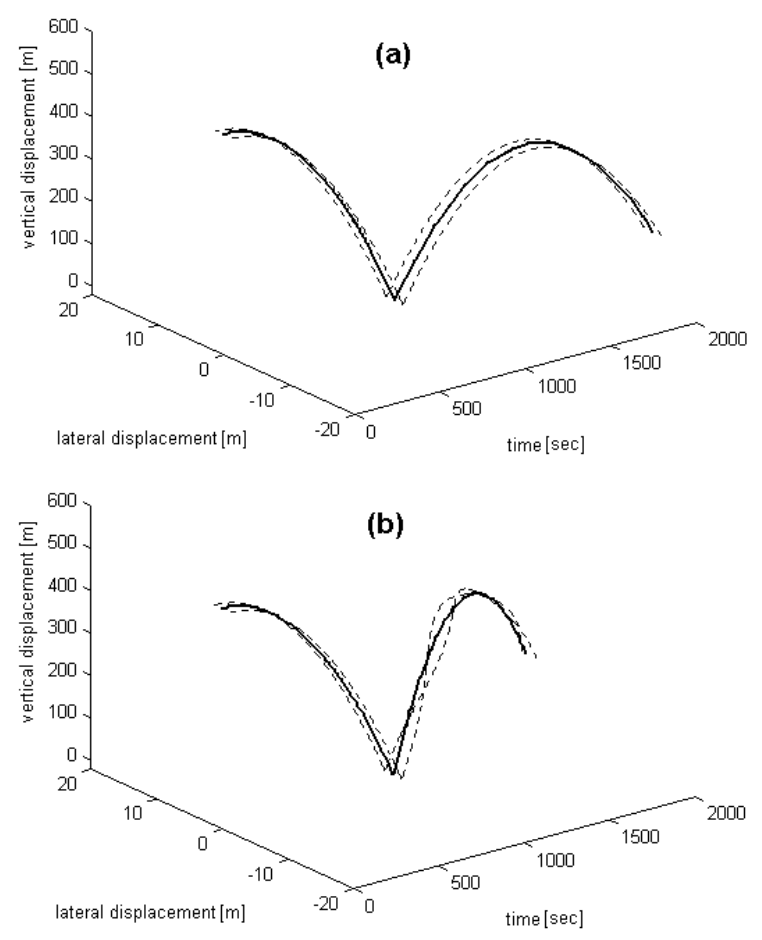

Figure 4: Example of descent and rebound

\section{Controllers}

\subsection{Phase-Plane Control}

The Phase-Plane (PP) controller that has been implemented here is an adaptation of a similar control algorithm that was used in the Apollo Lunar Module (LM) [6]. Fig.5 shows the logic for firing the thrusters. The parabolas in the figure are the switch curves that determine when the thrusters are turned on and off. During descent onto the asteroid surface the rate and the attitude of 
the lander are measured, and based on these measurements the thrusters are turned on/off.

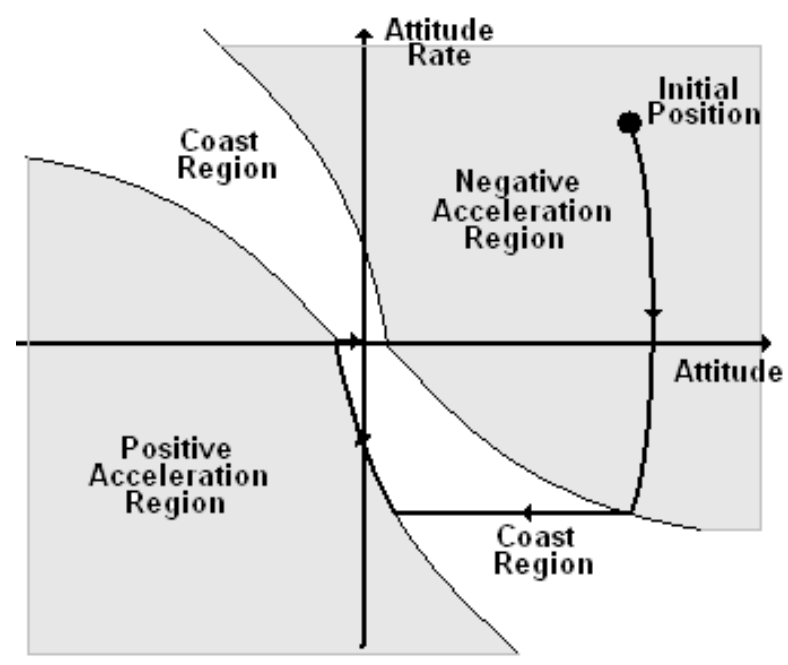

Figure 5: Phase plane switching logic

A relation is necessary to define the parabolas for the switching curves shown in Fig.5. Four such curves are needed, two for defining the points that the thrusters switch on and two that define the points that the thrusters switch off. The curve definitions are given below (equation (4)) and they are denoted in Fig.6.

$$
\begin{aligned}
\text { Fire }^{-} & =\theta+\frac{\dot{\theta}^{2}}{2 \cdot N \cdot \ddot{\theta}^{M a x}}-D B \\
\text { Fire }^{+} & =\theta-\frac{\ddot{\theta}^{2}}{2 \cdot N \cdot \ddot{\theta}^{M a x}}+D B \\
\text { Coast }^{-} & =\theta+\frac{\theta^{2}}{2 \cdot N \cdot \ddot{\theta}^{M a x}}+D B \\
\text { Coast }^{+} & =\theta-\frac{\ddot{\theta}^{2}}{2 \cdot N \cdot \ddot{\theta}^{M a x}}-D B
\end{aligned}
$$

where $\theta$ and $\dot{\theta}$ are the attitude and attitude rates respectively. $\mathrm{N}$ is the number of thrusters firing. $\ddot{\theta}^{M a x}$ is the acceleration produced from one thruster and DB is the magnitude of the dead-band.

The parameters specified in equation (4) will determine the shape of the parabolas depicted in Fig.6. The performance of the controller is therefore dominated by these parameters.

\subsection{Potential Function Control}

This controller uses the attitude and attitude rate of the lander to define an artificial potential function. The aim is then to minimise this potential as quickly as possible. This is done using Lyapunov's second method.

The aim of Lyapunov's second method is to guarantee the stability of a set of 


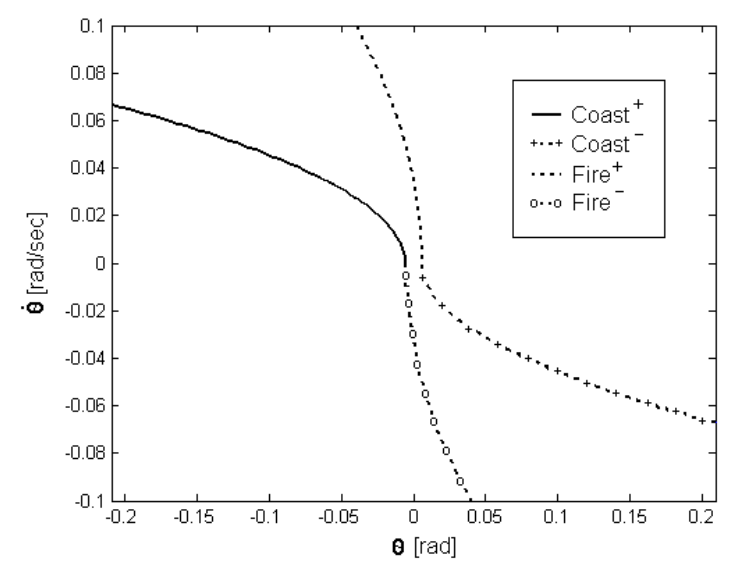

Figure 6: Phase plane

differential equations which describe a dynamical system. In physical terms, this has been described by Kalman and Bertram [13] [14].

The general form of the artificial potential is:

$$
V=f(\theta, \dot{\theta})
$$

The problem is to bring the lander to rest at some desired attitude so the terms to be controlled are the Euler angle $\theta$, and the body rate $\dot{\theta}$. The potential function, as defined in Radice [15] will be:

$$
V=V_{E U L E R}+V_{B O D Y R A T E}
$$

The component of the potential function due to the Euler angles will take the form of a quadratic polynomial function:

$$
V_{E U L E R}=\frac{1}{2}\left(\theta-\theta_{\text {Desired }}\right)^{n}, n=2,4,6, \ldots
$$

where $\theta_{\text {Desired }}$ is the goal attitude. The potential function due to the body rates will have a simpler form with the goal corresponding to null body rates:

$$
V_{B O D Y R A T E}=\frac{1}{2} \omega^{m}, m=2,4,6, \ldots
$$

where $\omega\left(=\left[\begin{array}{ll}0 & q\end{array}\right]^{T}\right.$, in this case) is the angular rate of the body.

The global potential, being the sum of the Euler and body rate components will therefore take the form:

$$
V=\frac{1}{2}\left(\theta-\theta_{\text {Desired }}\right)^{n}+\frac{1}{2} \omega^{m}
$$


To satisfy Lyapunov's theorem, the rate of change of the potential $\dot{V}$ must be rendered negative definite.

Therefore, differentiating the potential leads to:

$$
\begin{aligned}
\dot{V} & =\frac{n}{2}\left(\theta-\theta_{\text {Desired }}\right)^{n-1} \cdot \dot{\theta}+\frac{m}{2} \dot{\theta}^{m-1} \ddot{\theta} \\
& =\frac{n}{2}\left(\theta-\theta_{\text {Desired }}\right)^{n-1} \cdot \dot{\theta}+\frac{m M}{2 I} \dot{\theta}^{m-1}
\end{aligned}
$$

which will be used to generate the control law. A possible control torque M which will render $\dot{V}$ negative definite is expressed by:

$$
M=-\frac{\dot{\theta} \cdot I}{m \dot{\theta}^{m-1}} \cdot\left[2 \lambda \dot{\theta}+n \cdot\left(\theta-\theta_{\text {Desired }}\right)^{n-1}\right]
$$

where $\lambda$ is a positive definite shaping parameter for arbitrary $m$ and $n$. When the control torque is substituted into equation 10 the potential derivative then takes the form:

$$
\dot{V}=-\lambda \dot{\theta}^{2}
$$

so that the control law that rotates the lander to the desired goal attitude is available in analytical form. This solution to the control problem equates to a proportional derivative (PD) controller for $m=n=2$.

\subsection{Direct Adaptive Control}

Robust controllers try to desensitise a control system to the uncertainties of the plant. An adaptive controller aims to allow controller gains to vary continuously allowing for uncertainty and therefore increasing system performance during control implementation.

In [16], it was demonstrated that direct adaptive controllers can be applied to nonlinear oscillators. In [17], the concept was further proven in the use of electromagnetically controller oscillators and pneumatic cylinders. The concept has also been applied in other areas [18] [19].

Here, a direct adaptive control law is presented that provides robust attitude control of the asteroid lander. It is shown that the control law is a function of known system parameters and while being independent of external disturbances. $\mathrm{DAC}$ is therefore attractive for the asteroid lander problem since the mechanical properties of the asteroid surface will be highly uncertain.

\subsubsection{State Feedback for Uncertain Systems}

Consider the linear systems

$$
\dot{x}(t)=A \cdot x(t)+B \cdot u(t)+d
$$

with

$$
\mathbf{A}=\left[\begin{array}{c}
A_{0} \\
a
\end{array}\right], \mathbf{B}=\left[\begin{array}{c}
0_{\left(n_{x}-1\right) \times 1} \\
b
\end{array}\right], d=\left[\begin{array}{c}
0_{\left(n_{x}-1\right) \times 1} \\
d_{0}
\end{array}\right]
$$


where $x(t) \in \Re^{n_{x}}, u(t) \in \Re^{n_{u}}, d \in \Re^{n_{x}}, A_{0} \in \Re^{\left(n_{x}-1\right) \times n_{x}}, a \in \Re^{1 \times n_{x}}, b$, $d_{0} \in \Re$, and $b \neq 0$. Define $B_{0}=\left[\begin{array}{c}0_{\left(n_{x}-1\right) \times 1} \\ \operatorname{sign}(b)\end{array}\right]$

The following result is proven in [16].

Theorem. Assume there exists $K_{s} \in \Re^{n_{x}}$ such that $A_{s}=A+B . K_{s}$ is asymptotically stable. Let $R \in \Re^{n_{x} \times n_{x}}$ be positive semidefinite and assume $\left(A_{s}, R\right)$ is controllable. Let $P \in \Re^{n_{x} \times n_{x}}$ be the positive-definite solution to the Lyapunov equation $0=A_{s}^{T} \cdot P+P . A_{s}+R$. Finally, let $\Gamma \in \Re^{n_{u} \times n_{u}}$ and $\Lambda \in \Re^{n_{x} \times n_{x}}$ be positive definite, and let $\lambda>0$. Then the control law

$$
u(t)=\mathbf{K}(t) \cdot \mathbf{y}(t)+\phi(t)
$$

where

$$
\begin{gathered}
\dot{\mathbf{K}}(t)=-\Gamma \cdot \mathbf{B}_{0}^{T} \cdot P \cdot \mathbf{x}(t) \cdot \mathbf{x}^{T} \cdot \Lambda, \\
\dot{\phi}(t)=-\mathbf{B}_{0}^{T} \cdot P \cdot \mathbf{x}(t) \cdot \lambda
\end{gathered}
$$

yields $R \cdot \mathbf{x}(t) \rightarrow 0$ as $t \rightarrow \infty$ where $\mathbf{K} \in \Re^{n_{x}}$.

Note that equation (15) and equation (16) require the solution $P$ of the Lyapunov equation $0=A_{s} \cdot P+P . A_{s}+R$. Since $b \neq 0$, let $K_{s}=\frac{1}{b}\left(a_{s}-a\right)$, where $a_{s} \in \Re^{1 \times n_{x}}$. It follows that

$$
A_{s}=A+B \cdot K_{s}=\left[\begin{array}{c}
A_{0} \\
a
\end{array}\right]+\left[\begin{array}{l}
0 \\
b
\end{array}\right] \frac{1}{b}\left(a_{s}-a\right)=\left[\begin{array}{c}
A_{0} \\
a_{s}
\end{array}\right]
$$

Since $a_{s}$ can be chosen arbitrarily, it follows that $P$ can be determined without knowledge of $b$ or $a$. The only stipulation is that $\operatorname{sign}(b)$ must be known to implement equation (15) and equation (16).

To illustrate the theorem, consider the case $n_{x}=1$ and let $a_{s}<0$ and $R=-2 a_{s}$. Then $P=1$, and equation (15) and equation (16) are given by:

$$
\begin{gathered}
\dot{K}(t)=-(\operatorname{sign}(b)) \cdot \lambda_{1} \cdot x^{2}(t), \\
\dot{\phi}(t)=-(\operatorname{sign}(b)) \cdot \lambda_{2} \cdot x(t)
\end{gathered}
$$

where $\lambda_{1}=\Lambda / \Gamma$ and $\lambda_{2}=\lambda$. Note that equation (17) and equation (18) yield $x(t) \rightarrow 0$ as $t \rightarrow \infty$ for all $\lambda_{1}, \lambda_{2}>0$.

Next, consider the case $n_{x}=2$, and let $A_{0}=\left[\begin{array}{ll}0 & 1\end{array}\right], p>0, a_{s 1}<0, a_{s 2}<$ $-p$ and

Then

$$
R=\left[\begin{array}{cc}
-2 p a_{s 1} & 0 \\
0 & -2 p-2 a_{s 2}
\end{array}\right]
$$




$$
P=\left[\begin{array}{cc}
-p a_{s 2}-a_{s 1} & p \\
p & 1
\end{array}\right]
$$

satisfies $0=A_{s} \cdot P+P . A_{s}+R$ and equation (15) and equation (16) are given by

$$
\begin{aligned}
& \dot{K}_{1}(t)=-(\operatorname{sign}(b)) \cdot\left[\lambda_{1} p x_{1}^{2}(t)+\right. \\
& \left.\left(\lambda_{1}+\lambda_{12} p\right) x_{1}(t) x_{2}(t)+\lambda_{12} x_{2}^{2}(t)\right] \\
& \dot{K}_{2}(t)=-(\operatorname{sign}(b)) \cdot\left[\lambda_{12} p x_{1}^{2}(t)+\right. \\
& \left.\left(\lambda_{12}+\lambda_{2} p\right) x_{1}(t) x_{2}(t)+\lambda_{2} x_{2}^{2}(t)\right] \\
& \dot{\phi}(t)=-(\operatorname{sign}(b)) \cdot \lambda_{3}\left[p x_{1}(t)+x_{2}(t)\right]
\end{aligned}
$$

where

$\left[\begin{array}{cc}\lambda_{1} & \lambda_{12} \\ \lambda_{12} & \lambda_{2}\end{array}\right]=(1 / \Gamma) \Lambda$

is positive definite and $\lambda_{3}=\lambda>0$. Note that equation (19-21) yield $x(t) \rightarrow 0$ as $t \rightarrow \infty$ for all $p, \lambda_{1}, \lambda_{2}, \lambda_{3}>0$ and for all $\lambda_{12}$ such that $\lambda_{12}^{2}<\lambda_{1} \lambda_{2}$. Setting $\lambda_{12}=0$ for simplicity yields

$$
\begin{gathered}
\dot{K}_{1}(t)=-(\operatorname{sign}(b)) \lambda_{1}\left[p x_{1}^{2}(t)+x_{1}(t) x_{2}(t)\right] \\
\dot{K}_{2}(t)=-(\operatorname{sign}(b)) \lambda_{2}\left[x_{2}^{2}(t)+p x_{1}(t) x_{2}(t)\right] \\
\dot{\phi}(t)=-(\operatorname{sign}(b)) \cdot \lambda_{3}\left[p x_{1}(t)+x_{2}(t)\right]
\end{gathered}
$$

The above theorem can be applied to the following general control problem. Consider the $n$ th-order linear system:

$$
r^{n}(t)-a_{n} r^{n-1}(t)-\cdots-a_{2} \dot{r}(t)-a_{1} r(t)=b u(t)
$$

with the requirement that $r(\mathrm{t})$ approaches $r_{\text {des }}$ without knowledge of $a=$ $\left[a_{1} \cdots a_{n}\right]$ and $b$, except for the sign of $b$. Defining the error signal $x_{1}(t) \triangleq$ $r(t)-r_{\text {des }}$ and the state $x=\left[\begin{array}{llll}x_{1} & \dot{x}_{1} & \cdots & x_{1}^{n-1}\end{array}\right]^{T}$, equation (25) becomes:

$$
\dot{x}(t)=A \cdot x(t)+B \cdot u(t)+\left[\begin{array}{c}
0_{(n-1) \times 1} \\
d_{0}
\end{array}\right]
$$

where $A=\left[\begin{array}{c}A_{0} \\ a\end{array}\right]$ and $d_{0}=-a_{1} r_{d e s}$, where $A_{0}=\left[0_{(n-1) \times 1} I_{n_{x}-1}\right], a=$ $\left[a_{1} \cdots a_{n}\right]$, and $I_{n_{x}-1}$ is the $\left(n_{x}-1\right) \times\left(n_{x}-1\right)$ identity matrix. Thus equation (26) has the form of equation (13). Note that $d_{0}$ is unknown since $a_{1}$ is unknown. Thus the controller in equation $(14-16)$ can be used for the lander attitude control problem. 


\subsubsection{State Feedback for Lander Attitude Control}

The theorem defined above can now be applied to the attitude control problem. Consider the lander attitude dynamics modeled by:

$$
\ddot{\theta}(t)=M / I
$$

where and $M$ is the sum of all the torques acting on the body, including the control torque $u(t)$ and the disturbing torque $d$ which is defined by the moment resultant on the lander as a result of a difference in the force acting on the base from the individual legs. $I$ is the moment of inertia of the body about the axis of rotation, and so:

$$
M=u+d
$$

The control objective is to require $\theta$ to approach $\theta_{\text {des }}$ without knowledge of $d$. Defining the error signal $x_{1}(t)=\theta(t)-\theta_{\text {des }}$ and the state $\mathbf{x}=\left[\begin{array}{ll}x_{1} & \dot{x}_{1}\end{array}\right]^{T}$, equation (27) becomes:

$$
\dot{\mathbf{x}}(t)=\left[\begin{array}{ll}
0 & 1 \\
0 & 0
\end{array}\right] \mathbf{x}(t)+\left[\begin{array}{l}
0 \\
b
\end{array}\right] u(t)+\left[\begin{array}{l}
0 \\
d
\end{array}\right]
$$

Now solving Lyapunov's equation using the general solutions to $R$ and $P$ maintaining $A_{s}$ asymmetrically stable gives the variable control gains to the problem as:

$$
\begin{gathered}
\dot{K}_{1}(t)=-\left(1.5 \theta^{2}+2 \theta \dot{\theta}\right) \\
\dot{K}_{2}(t)=-\left(1.5 \theta \dot{\theta}+2 \dot{\theta}^{2}\right) \\
\dot{\phi}(t)=-(0.1 \theta+10 \dot{\theta})
\end{gathered}
$$

This in turn leads to the implied control moment as:

$$
u(t)=\left(K_{1} \theta+K_{2} \dot{\theta}+\phi\right) . I
$$

The advantage of using this control algorithm is due to the fact it can react in real time to any disturbing moment, in this case $d$. Due to the unknown composition of the asteroid the contact dynamics are unknown and the DAC controller is best placed to deal with these uncertainties.

\section{Descent in a Rotational Frame}

The Macro-Polo lander will be required to land at a specified point on the surface of the asteroid. A rotational reference [20] frame has been chosen for kinematic modeling of the descent to ensure the lander remains in the correct 
lateral position during descent. This introduces an extra requirement on the lander for lateral control during descent.

Fig.7 depicts the difference between an inertial and rotating frame of reference during the lander descent. From an initial rotation position $\phi$ and with a constant reference frame rotation rate $\omega=\left[\begin{array}{lll}0 & \mathrm{q} & 0\end{array}\right]$, the inertial frame will see the lander spiral and the rotating frame will see the lander descend directly towards the surface in a straight line. The spacing between the points on each line represent equal time intervals.

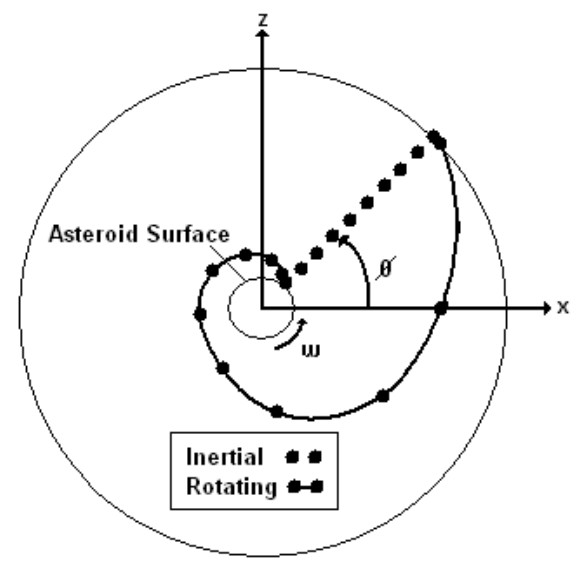

Figure 7: Reference frame comparison

The outcome of this is the introduction of fictitious forces acting on the lander as it descends in the rotating frame.

$$
\mathbf{F}_{\text {fict }}=-2 m \omega s \mathbf{u}_{\theta}+m \omega^{2} R(t) \mathbf{u}_{R}
$$

where $m$ is the mass of the lander, $\omega=\left[\begin{array}{lll}0 \mathrm{q} & 0\end{array}\right], s$ is the descent velocity, $R$ is the radial position of the lander, and $\mathbf{u}_{R}$ and $\mathbf{u}_{\theta}$ are unit vector in the radial and tangential direction respectively.

To obtain straight line motion in the rotating frame, a force exactly opposite to the fictitious force must be applied to reduce the net force on the lander to zero, for straight line motion in agreement with the rotational frame. The fictitious forces that must be nulled are the Coriolis force (first term in equation (34)) and the centrifugal force (second term in equation (34)).

With respect to the lander model these two forces can be represented as:

$$
\begin{gathered}
F_{C O R}=-2 m \omega \times \mathbf{V} \\
F_{C E N}=-m \omega \times(\omega \times \mathbf{P})
\end{gathered}
$$

where $\mathbf{V}=(u, 0, w)$ and $\mathbf{P}=(x, 0, z)$. 
The process of introducing these new forces will result in the lander appearing to descend in a straight line towards the surface of the asteroid although in reality it will curve around the surface of the asteroid with angular velocity $\omega$.

Introducing a DAC control law for lateral displacement using the same principles as for the rotational control maintains that the lander will be in the correct position as it approaches the asteroid surface. Drawing comparison with equation (27) and equation (29) produces:

$$
\ddot{x}(t)=T_{\text {lat }} /\left(m_{B}+2 . m_{L}\right)
$$

and

$$
\dot{\mathbf{x}}(t)=\left[\begin{array}{ll}
0 & 1 \\
0 & 0
\end{array}\right] \mathbf{x}(t)+\left[\begin{array}{l}
0 \\
b
\end{array}\right] u(t)+\left[\begin{array}{l}
0 \\
d
\end{array}\right]
$$

where, $\mathbf{x}=\left[\begin{array}{ll}x_{1} & x_{2}\end{array}\right]=[\dot{x} x], T_{\text {lat }}$ is the lateral force produced by the thrusters, $m_{B}+2 . m_{L}$ is the total spacecraft mass, $x_{2}(t)=x-x_{d e s}$ is the error signal and is the displacement $x$ from the desired landing location $x_{d e s}$. Following the earlier analysis a similar control algorithm is produced:

$$
\begin{gathered}
\dot{K}_{1}(t)=-\left(1.5 x_{2}^{2}+2 x_{2} \dot{x_{2}}\right) \\
\dot{K_{2}}(t)=-\left(1.5 x_{2} \dot{x_{2}}+2 \dot{x_{2}}{ }^{2}\right) \\
\dot{\phi}(t)=-\left(0.1 x_{2}+10 \dot{x_{2}}\right)
\end{gathered}
$$

Subsequently leading to the necessary lateral control thrust:

$$
T_{l a t}=\left(K_{1} x_{2}+K_{2} \dot{x_{2}}+\phi\right) \cdot\left(m_{B}+2 m_{L}\right)
$$

With regards the lateral control the disturbance $d$ in equation (38) is now comprised of the coriolis and centrifugal forces defined in equation (35) and equation (36) in addition to any translational forces that may be induced by the control thrusters minimising the attitude error, due to the fact this is done in body axes.

\section{Results for Controlled Descent}

Having a model and three controllers in place a comparative simulation can be done to asses the performance of the controllers against one another. The first step in doing so is to define a performance parameter that can be used as a basis for comparison. The performance parameter selected in this instance is the total impulse used by the lander system to bring the lander to rest on the surface of the asteroid. Total impulse is defined as:

$$
I_{T}=\sum_{i=1}^{n} \Delta t . T
$$


where $\Delta t$ is the integration step magnitude, $T$ is the corresponding thrust level at the integration interval and $n$ is the number of integration steps. It is now possible to develop a comparison between the three controllers.

The descents and rebounds will take place in a rotational frame of reference as depicted in Fig.3 and the equations of motion will be those outline by equation (1). Finally, a simple bounce alleviation algorithm is defined: if the attitude of the lander is within a small error limit $\left(\theta_{\text {des }} \pm 0.5\right.$ degrees $)$ and the vertical velocity of the lander is negative (i.e. ascending) then two thrusters are fired in a positive vertical sense (in body axes) to reduce any residual bounces.

Before a direct comparison can be made nominal values must be selected for the controller constants in each controller. For the potential function controller this is a single shaping parameter, $\lambda$, and the thruster threshold ( 0.1 Newtons). The thruster threshold defines the lower limit of necessary demanded thrust before the thruster is switched on, this eliminates any unwanted chattering by the thrusters. For the Phase Plane controller there are two parameters: $\ddot{\theta}^{\text {Max }}$ and DB described earlier.

For the purpose of this comparison, for each lander descent scenario, the control parameters have been optimised using a simple trial and error procedure. Therefore, an approximate optimum value for the total impulse in each case has been established.

There also exists a number of other scenario parameters. These are specified below in table 2 and are common to all the lander descent simulations. This data was supplied by Astrium Ltd for the Marco-Polo mission [12].

Table 2: Scenario parameters

\begin{tabular}{|c|c|}
\hline Mass of Base $(\mathrm{kg})$ & 985.4 \\
\hline Mass of Leg $(\mathrm{kg})$ & 7.3 \\
\hline Inertia of Lander $\left(\mathrm{kg} . \mathrm{m}^{2}\right)$ & 677 \\
\hline Gravitational Accel $\left(\mathrm{m} / \mathrm{s}^{2}\right)$ & 0.000145 \\
\hline Asteroid rotational Rate $(\mathrm{deg} / \mathrm{sec})$ & 0.013 \\
\hline Initial Inertial Position $([\mathrm{m}, \mathrm{m}])$ & {$[0,1000]$} \\
\hline$\theta_{\text {Desired }}(\mathrm{rad})$ & 0 \\
\hline Minimum Impulse Bit $(\mathrm{sec})$ & 0.005 \\
\hline Leg Stroke Length $(\mathrm{m})$ & 0.3 \\
\hline Asteroid Radius $(\mathrm{m})$ & 500 \\
\hline
\end{tabular}

The scenarios selected for the comparison each have a differing starting attitude. In each case the lander starts at $[0,1000] \mathrm{m}$ as specified in table 2 . The lander initial attitude is then specified as 10, 20 or 30 degrees and permitted to descend towards the asteroid.

Optimisation of the control parameters for each controller in each case gives the values specified in table 3 . In the case of the PF and DAC controllers a thruster threshold is included to reduce chattering and is assigned to be 0.01 Newtons. In the case of the PP controller the dead band value is selected to be 0.0025 radians. An optimal solution for the three initial states of the DAC 
controller were found to be: $\kappa_{1}(0)=0.0, \kappa_{2}(0)=-2.75$ and $\phi_{1}(0)=0.0$, for all three initial attitude states again demonstrating the robustness of DAC.

Table 3: Controller optimised parameters

\begin{tabular}{|c|c|c|}
\hline $\begin{array}{c}\text { Initial Attitude } \\
(\mathrm{deg})\end{array}$ & $\begin{array}{c}\mathrm{PF} \\
\lambda\end{array}$ & $\begin{array}{c}\mathrm{PP} \\
\ddot{\theta}^{M a x}\end{array}$ \\
\hline 10 & 1.8 & 0.0035 \\
\hline 20 & 2.35 & 0.0035 \\
\hline 30 & 2.8 & 0.0035 \\
\hline
\end{tabular}

The values for total impulse calculated in the scenarios described above are given in table 4 . The interesting result that appears is when the Potential Function controller corrects the 30 degree initial attitude error more efficiently that the DAC algorithm. This result is explained by analysis of the transient correction time necessary in the manoeuvre, given in table 5 . It follows that the DAC controller, in the 30 degree case, takes slightly longer than the actual descent time of the lander to correct the error. Control is hence carried through the contact phase and into the rebound resulting in a considerable increase in impulse necessary.

Table 4: Total impulse for descent scenarios

\begin{tabular}{|c|c|c|c|c|}
\hline \multicolumn{2}{|c|}{ Scenario } & $\begin{array}{c}\text { PP } \\
\text { Impulse } \\
\text { (N.sec) }\end{array}$ & $\begin{array}{c}\text { PF } \\
\text { Impulse } \\
\text { (N.sec) }\end{array}$ & $\begin{array}{c}\text { DAC } \\
\text { Impulse } \\
\text { (N.sec) }\end{array}$ \\
\hline \multirow{3}{*}{$\theta_{i}=10^{0}$} & Rot & 14.08 & 5.98 & 1.79 \\
& Trans & 272 & 647 & 183 \\
& $\Sigma$ & 286.08 & 652.98 & 184.79 \\
\hline \multirow{3}{*}{$\theta_{i}=20^{0}$} & Rot & 18.05 & 8.26 & 4.86 \\
& Trans & 290 & 666 & 195 \\
& $\Sigma$ & 308.05 & 674.26 & 199.86 \\
$\theta_{i}=30^{0}$ & Rot & 21.56 & 19.07 & 21.67 \\
& Trans & 311 & 685 & 241 \\
& $\Sigma$ & 332.56 & 704.07 & 262.67 \\
\hline
\end{tabular}

A different result to that of the rotational control is found in the joint rotational and translational study. Here, the thrusters are used to compensate for the coriolis force and ensure a vertical descent. The magnitude of the impulse required to control the lander in translational descent being largest for the potential function controller and smallest for the direct adaptive controller with the phase plane magnitude somewhere between the two results. As with the rotational control each controller is capable of maintaining translational control but the DAC algorithm is the most efficient.

The difference between the results for the two studies (rotational and trans- 
Table 5: Settling times for descent scenarios

\begin{tabular}{|c|c|c|c|}
\hline $\begin{array}{c}\text { Initial } \\
\text { Attitude } \\
(\mathrm{deg})\end{array}$ & $\begin{array}{c}\text { Settling } \\
\text { Time } \\
(\mathrm{sec}) \\
\mathrm{PP}\end{array}$ & $\begin{array}{c}\text { Settling } \\
\text { Time } \\
(\mathrm{sec}) \\
\text { PF }\end{array}$ & $\begin{array}{c}\text { Settling } \\
\text { Time } \\
(\mathrm{sec}) \\
\text { DAC }\end{array}$ \\
\hline 10 & 6.9 & 19.2 & 820 \\
\hline 20 & 9.9 & 16.2 & 840 \\
\hline 30 & 12.4 & 20.1 & 1010 \\
\hline
\end{tabular}

lational) can be explained by the dynamics required to rectify the control error. This time the error is the lateral displacement from the desired position. In the attitude case the aim is to correct a step error input, but in the translational case the error is continually changing due to the continuous rotation of the asteroid. The DAC algorithm is capable of reacting to both cases due to a real-time change in its control parameters. The Phase-Plane and Potential Function controllers contain constants and these are optimised to a specific scenario, leading to a greater impulse as the time for the descent increases. An example plot of a descent and rebound scenario can be seen in Fig.8. Here no initial attitude error is included but the bounce alleviation algorithm can be seen to be working efficiently. An attitude correction simulation can be seen in Fig. 9 - this is a detailed plot of the first 20 seconds of descent as in most descent scenarios the attitude of the lander is corrected rapidly. The black frame in Fig. 9 are a representation of the lander.

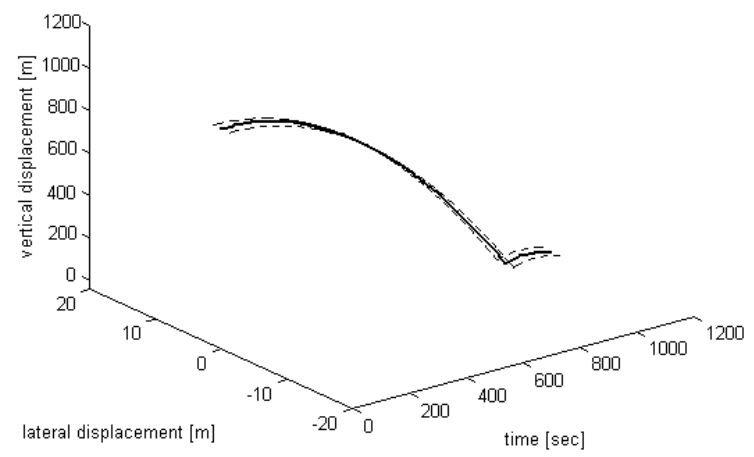

Figure 8: Example of descent and rebound

\section{$7 \quad$ FDIR Studies}

Space science missions involving autonomous landing capabilities have many unknown aspects. Examples of these are exact topological information of the 


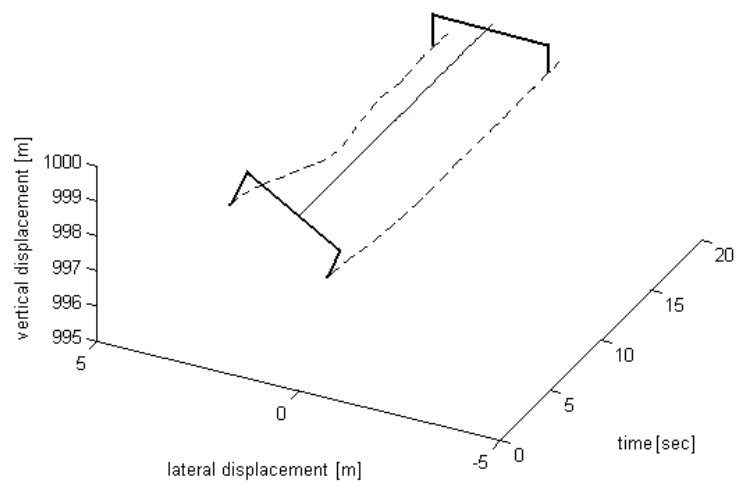

Figure 9: Attitude correction

landing site and the composition of the asteroid upon which the landing is to be made. If an unexpected event were to arise during descent or during science operations on the asteroid it is necessary for the system to have the ability to react. To this end it is desirable to have some degree of Fault detection, Isolation and Recovery (FDIR) embedded into the system to minimize the chance of mission failure [21].

\subsection{Lander Leg Failures}

The first of the studies into the FDIR properties of the three controllers is a comparison of the ability of the controllers to recover from three differing leg failure scenarios. Firstly one of the two legs is induced to fail by becoming overly 'stiff' with regards to the other leg. The failing leg is assumed to have a doubling of the spring constant in that particular leg leading to a consequent imbalance between the two legs. Similarly to the first case, the second failure scenario is when one of the lander leg spring stiffness constants is half the other. Finally, the third failure scenario is when one leg is artificially 'stuck', where no movement is permitted in this leg.

Ideally the lander is required to settle on the surface of the asteroid using as little propellant as possible while maintaining a small attitude error. An example of the initial descent, contact and first rebound phase of the scenario is given in Fig.10. The insert shows a close up of the contact phase. Also, the black frames show a representation of the attitude of the lander as the simulation progresses. During contact the insert shows that the lander attitude increases and the control law is capable of returning the lander to zero attitude error before the next contact with the asteroid occurs.

The initial conditions for the simulations are the same as for the non-failure scenarios. The lander simulation is started at a height of $500 \mathrm{~m}$ above the 
surface of a $500 \mathrm{~m}$ radius asteroid. The lander has an initial lateral velocity corresponding to the rotational rate of the asteroid at a $1000 \mathrm{~m}$ radial distance. The aim of the simulation is to land at rest with zero attitude and lateral displacement error.

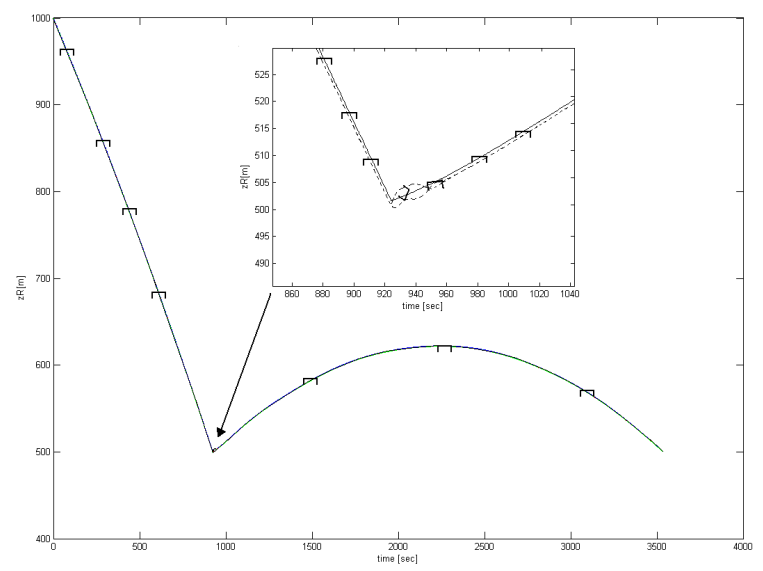

Figure 10: Scenario example using a conventional controller

For analysis there are two properties of the simulation that will be used for comparison. As before the total impulse of the scenario will be used to compare the propellant necessary to recover from each of the leg failures. Another property of interest here is the maximum attitude that is induced by the leg failures. This property is representative of the ability of the lander to maintain the necessary track on the asteroid surface for such instrumentation as radar/LASER altimetry [22] as this instrumentation will be positioned on the lander such that it takes readings in the positive vertical body axis direction.

The results for the study are shown in table 6 and Fig.11 - 13, where the contact dynamics are evident at approximately $880 \mathrm{~s}$. The relative attributes of the different controllers now begin to become more apparent. As was evident with the two previous studies, without disturbances, qualitatively the algorithms have inherent benefits/weaknesses. The DAC algorithm is in general the most effective.

Evaluating the evolution of the attitude during the controlled descent demonstrates the weaknesses of the phase plane and potential function controllers with the maximum overshoots being larger that the DAC results. In all cases the controllers are capable of maintaining control of the lander. However, an overshoot of over 90 degrees is clearly too extreme for the altimetry to continue to function and hence the potential function and phase plane controllers may be deemed to fail in some cases. 


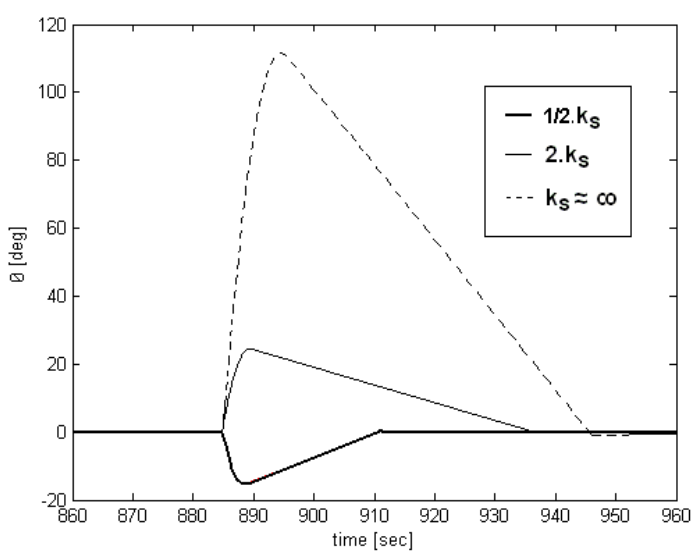

Figure 11: $\theta$ for $\mathrm{PF}$

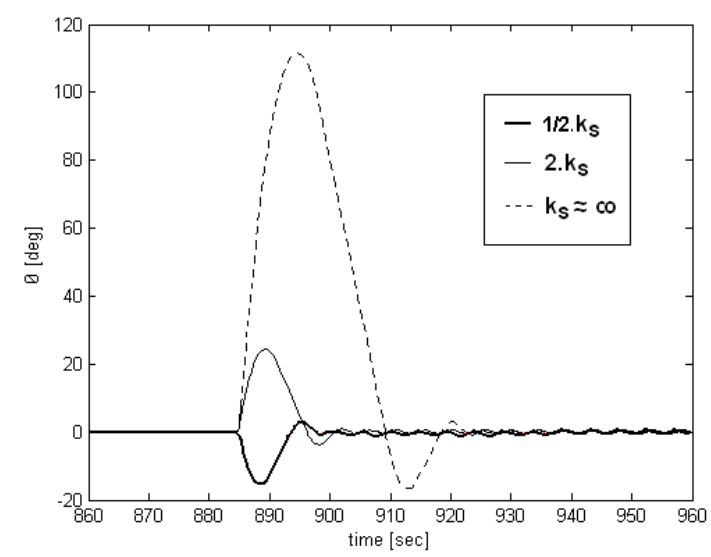

Figure 12: $\theta$ for $\mathrm{PP}$ 
Table 6: Impulses for Leg Failure Scenarios

\begin{tabular}{|c|c|c|c|c|}
\hline \multicolumn{2}{|c|}{ Scenario } & $\begin{array}{c}\text { PP } \\
\text { Impulse } \\
\text { (N.sec) }\end{array}$ & $\begin{array}{c}\text { PF } \\
\text { Impulse } \\
\text { (N.sec) }\end{array}$ & $\begin{array}{c}\text { DAC } \\
\text { Impulse } \\
\text { (N.sec) }\end{array}$ \\
\hline \multirow{3}{*}{$k_{s} \times 0.5$} & Rot & 852 & 871 & 653 \\
& Trans & 995 & 1942 & 810 \\
& $\Sigma$ & 1847 & 2813 & 1463 \\
\hline \multirow{3}{*}{$k_{s} \times 2$} & Rot & 850 & 878 & 654 \\
& Trans & 1237 & 2022 & 900 \\
& $\Sigma$ & 2115 & 2872 & 1554 \\
\hline \multirow{3}{*}{$k_{s} \approx \infty$} & Rot & 2062 & 1767 & 1880 \\
& Trans & 2623 & 6017 & 930 \\
& $\Sigma$ & 4686 & 7784 & 2810 \\
\hline
\end{tabular}

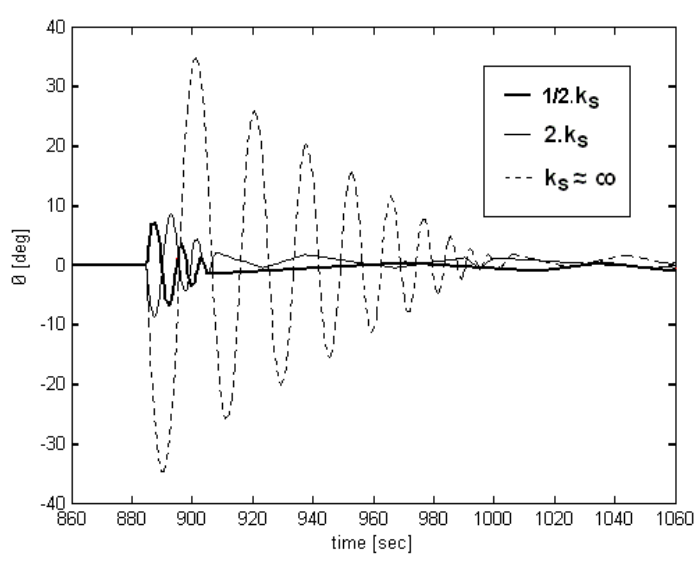

Figure 13: $\theta$ for DAC

These large overshoots will also contribute to extra necessary translational control. As the attitude of the lander increases any corrective control will contribute to the height of the rebound. The higher this rebound the more time the lander will spend correcting for translational errors leading to greater impulse requirements.

\subsection{Lander Thruster Failure}

The second FDIR scenario to be investigated is when one of the thrusters used for rotational control is failed. During this study we will consider two modes of thruster failure. Firstly when one thruster (of the four) becomes inoperable - this leaves only one thruster for control in one rotational sense and the usual two thrusters for control in the opposing direction. Secondly, the case where 
one thruster continuously fires - leaving the lander in a continual spin unless suitable corrective thrust is applied. Referring to Fig. 2 it will be thruster 1 that will be assumed to fail in both cases. A comparison of the three controllers leads to an another insight into the FDIR capabilities.

Thruster 1 is failed and a study similar to that described in section 7.1 is performed. The same initial conditions and constants are used for both the studies, although only the 10 degree initial attitude case is under investigation here.

Tables 7 and 8 show a comparison of the impulse and settling times required for this failure induced study. Interesting results appear in the tables. It appears that there is no large apparent change in the magnitude of impulse required to control the lander, indeed in the cases of all control algorithms there is no significant gain to be made.

Table 7: Total impulse for thruster failures

\begin{tabular}{|c|c|c|c|}
\hline $\begin{array}{c}\text { Initial } \\
\text { Attitude } \\
(10 \mathrm{deg})\end{array}$ & $\begin{array}{c}\text { Total } \\
\text { Impulse } \\
\text { (N.sec) } \\
\mathrm{PP}\end{array}$ & $\begin{array}{c}\text { Total } \\
\text { Impulse } \\
(\mathrm{N} . \mathrm{sec}) \\
\mathrm{PF}\end{array}$ & $\begin{array}{c}\text { Total } \\
\text { Impulse } \\
\text { (N.sec) } \\
\text { DAC }\end{array}$ \\
\hline No Failure & 14.078 & 5.98 & 1.7898 \\
\hline Off Failure & 14.299 & 5.84 & 1.461 \\
\hline On Failure & 14.418 & 6.16 & 2.005 \\
\hline
\end{tabular}

In table 7 the impulse used by the thruster which is continuously firing has been neglected so an easier comparison can be drawn. The numerical values for the impulse for the continuously firing thruster in the three simulation are: PP - 8923 [N.sec], PF - 8957 [N.sec], DAC - 8998 [N.sec].

Table 8: Settling times for thruster failures

\begin{tabular}{|c|c|c|c|}
\hline $\begin{array}{c}\text { Initial } \\
\text { (10 deg) }\end{array}$ & $\begin{array}{c}\text { Settling } \\
\text { Time } \\
(\mathrm{sec}) \\
\mathrm{PP}\end{array}$ & $\begin{array}{c}\text { Settling } \\
\text { Time } \\
(\mathrm{sec}) \\
\mathrm{PF}\end{array}$ & $\begin{array}{c}\text { Settling } \\
\text { Time } \\
(\mathrm{sec}) \\
\text { DAC }\end{array}$ \\
\hline No Failure & 6.9 & 19.2 & 820 \\
\hline Off Failure & 10.1 & 21.1 & 850 \\
\hline On Failure & 10.3 & 21.0 & 847 \\
\hline
\end{tabular}

An explanation for these slight changes in impulse can be attributed to the model being a freely rotating rigid body with no resistance to rotation. The smaller thrust induced by the failure allows fine-tuning of the attitude to be done easier, although over a slightly longer time period. Both these traits can be seen on analysis of Fig.14 that depicts the DAC attitude error. Here the longer settling times are associated with smaller individual error correcting impulses.

The increase in settling time can be seen in Fig. 15 that depicts the attitude error for each of the failure scenarios during the course of the potential function 
decrease. This increase in settling time in the off failure case is due to only one thruster being available for negative rotation. It is then evident that the on failure case only starts to differ from the no failure case when positive rotation is required. Due to the continuous negative thruster failure the result differs slightly when in the positive rotational sense.

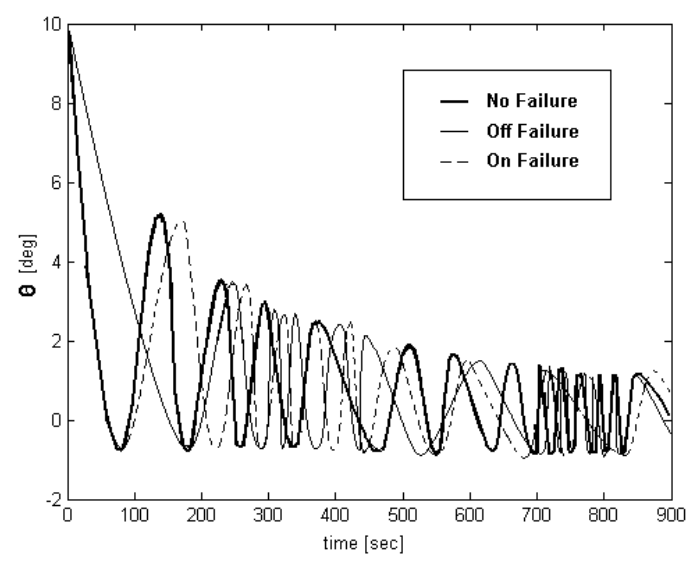

Figure 14: Attitude error for DAC

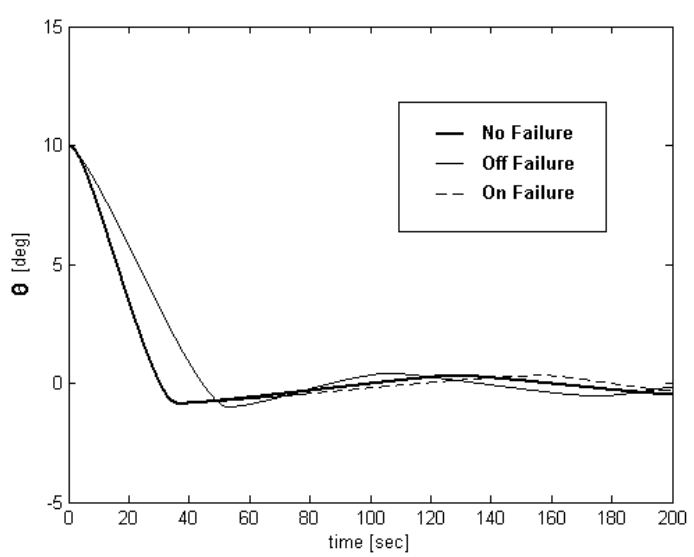

Figure 15: Attitude error for potential function

Finally to explain the slight increase in impulse and settling time in the phase-plane cases Fig.16 shows the attitude error. The longer time periods spent passing each end of the dead-band accompanied by the longer settling time lead to the total impulse being slightly larger. 


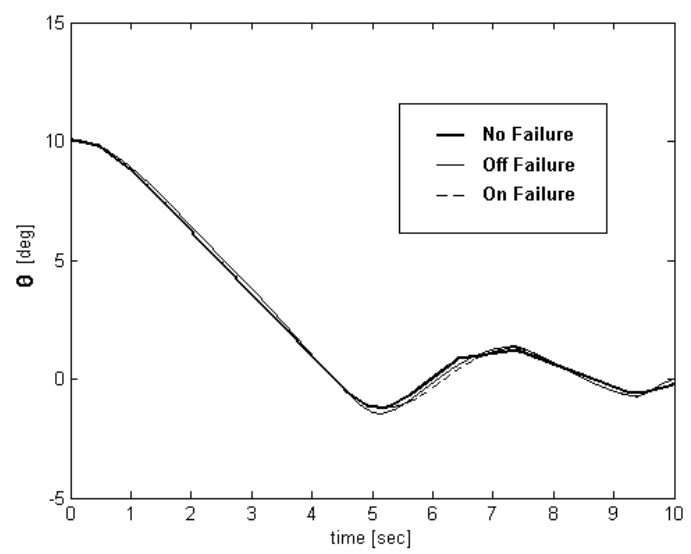

Figure 16: Attitude error for phase-plane

\section{Sampling Study}

In addition to the attitude/rotational control and FDIR studies performed above, an additional study has been performed to indicate propellant gains that can be made during the asteroid sampling process by adopting a DAC algorithm. The control force in this instance is intended to be used as a alternative mechanism for maintaining contact with the asteroid during the sampling process. The proposed method for maintaining contact with the surface is a continuous thrust towards the asteroid using two or more cold gas thrusters [23].

The aim of this simulation is to stabilise the lander at the stable position the lander rests at after descent. Any deviation from this desired vertical location induces an error into the control law and this error drives the thrusters. A schematic of the system under analysis is given in Fig.17.

To model the unknown composition of the asteroid a varying reaction drill force is introduced. The maximum reaction force is limited at ten times the weight of the lander base. Also, the reacting force is permitted to vary its form; firstly the reaction force follows a sinusoidal path from maximum to zero and return, then an intermittent pulsing force with discontinuous steps from maximum to zero force is introduced. By doing this most of the possible surface materials that may be encountered are modeled. In these cases: a solid rock (constant force), a porous material (sinusoid force), and a solid with voids (pulsed force).

Visualization of the control process is demonstrated with a plot of the required reaction force in Fig.18(a) with a plot of the produced thrust in Fig.18(b) below. The impulse required for this simulation is given in table 9 alongside the theoretical impulse that would be required for the base-line approach.

Evidently the impulse required to stabilise the lander is much less using the DAC algorithm compared to the classical approach. The reason for this 
becomes apparent on analysis of Fig.18. Due to the varying type of reaction force that is encountered during the sampling process it is not always necessary to continually counteract the reaction force. The DAC algorithm is capable of selecting the appropriate times when a control thrust is not needed, leading to savings in the total impulse and required propellant mass.

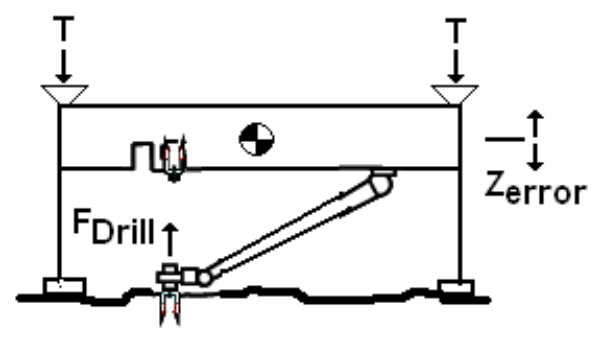

Figure 17: Sampling
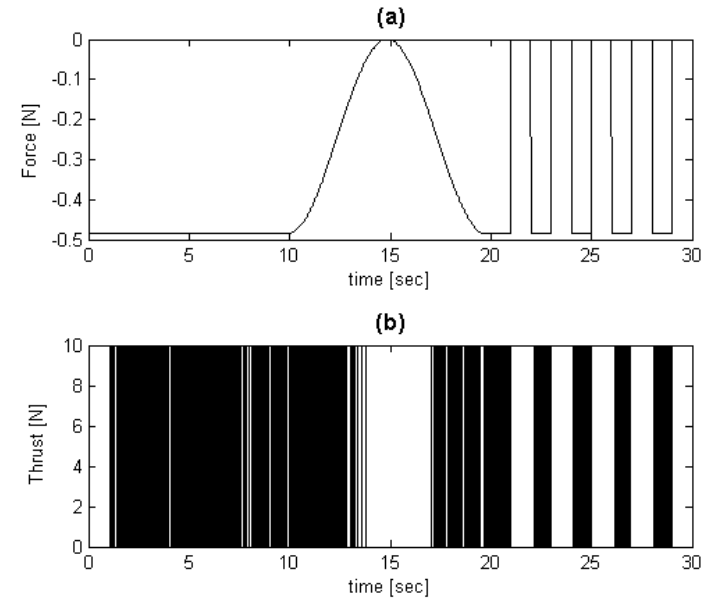

Figure 18: Reaction force (a), thrust force (b)

\section{Conclusions}

The different studies conducted in this paper demonstrate the relative advantages to be gained from appropriate selection of controllers. Throughout the paper the DAC method of control continually out performs in all areas except that of reaching a settled state in a small time period. However, the argument for the decrease in impulse required for each of these studies implying weight 
Table 9: Total impulse for stabilization

\begin{tabular}{|c|c|c|}
\hline & Classical & DAC \\
\hline $\begin{array}{c}\text { Total } \\
\text { Impulse } \\
\text { (N.sec) }\end{array}$ & 600 & 46 \\
\hline
\end{tabular}

savings in the overall propellant budget required for mission greatly outweighs this.

This paper demonstrates through simulation that an evolutionary process of moving to a 'bottom-up' internally integrated method of autonomous control will have several advantages over classical systems. Firstly through propellant mass savings, secondly through better tracking by minimising attitude errors, and thirdly introducing autonomous control algorithms that can be mathematically verified.

\section{Acknowledgements}

This work was sponsored by the Science and Technology Facilities Council (STFC) and EADS Astrium UK Ltd through a CASE award (Blair Brown) and European Research Council grant VISIONSPACE 227571 (Colin McInnes).

\section{References}

[1] Faye F Villefrache P, Evans J. Rosetta: The esa comet rendevous mission. Acta Astronautica, 40:871-877, 1997.

[2] Michael R. Combi Michael F. AHearn. Deep impact at comet tempel 1. ICARUS, 0019-1035, 2007.

[3] Tatsuaki H Takashi K, Masatsugu O. Touchdown dynamics for sample collection in hayabusa mission. In 2008 IEEE International Conference on Robotics and Automation, Pasadena, May 19-23 2008.

[4] Ichiro N. Tetsua Y., Takashi K. Hopping rover 'minerva' for asteroid exploration. In Proc. Fifth International Symposium on Artificial Intelligence, number ESA SP 440, ESA, June 1-3 1999.

[5] Ulamec S. Biele J. Capabilities of philae, the rosetta lander. Space Science Review, 138:275-289, 2008.

[6] Widnall W. S. Lunar module digital autopilot. Journal of Spacecraft and Rockets, 8:56-60, 1970.

[7] Sembely X. Lignc summary report. EADS Astrium, EEA.TCN.97388.ASTR, 2005. 
[8] Mukherji R. Allen A., Langley C. Full-scale testing and platform stabilization of a scanning lidar system for plantery landing. In Conference on Space Exploration Technologies, Orlando, March 2008.

[9] Tripp J. Lafontaine J. Ulitsky A. Laps: The development of a scanning lidar system with gnc for autonomous hazard avoidance and precision landing. In Conference on Spaceboard Sensors, Orlando, April 2004.

[10] Della Torre A. Guizzo G.P., Bertoli A. Testing gnc technologies for planetary landing on mars and moon analogues. In 2nd International Workshop - Exploring Mars and its Earth Analogues, Trento, June 2007.

[11] Della Torre A. Guizzo G., Bertoli A. Mars and moon exploration passing through the european precision landing gnc test facility. In 58th International Astronautical Congress, Hyderabad, India, September 2007.

[12] Yoshikawa M. Michel P. Barucci M. Marco polo: near earth object sample return mission. Experimental Astronomy, 23:785-808, 2009.

[13] Bertram R. E. Kalman R. E. Control system analysis and design via the second method of lyapunov part i: Continuous systems. ASME, 83:371-393, 1960 .

[14] Bertram R. E. Kalman R. E. Control system analysis and design via the second method of lyapunov part ii: Discrete systems. ASME, 83:394-400, 1960.

[15] Radice G. Behavior Based Autonomy for Single and Multiple Spacecraft. PhD thesis, University of Glasgow, 2002.

[16] Bernstein D. S. Hong J. Adaptive stabilization of non-linear oscillators using direct adaptive control. Internation Journal of Control, 74:432-444, 2000 .

[17] Bernstein D. S. Hong J., Cummings I. A. Experimental application of direct adaptive control laws for adaptive stabilization and command following. In Conference on Decision and Control, number TuM11-1440, Pheonix, December 1999.

[18] Houjun T. Yimei C., Zhengzhi H. Direct adaptive control for nonlinear uncertain system based on control lyapunov function method. Journal of Systems Engineering and Electronics, 17:619-623, 2006.

[19] McInnes C. R. Direct adaptive control for gravity-turn descent. Journal of Guidance, 22:374-377, 1998.

[20] Taylor J. Classical Mechanics. University Science Books, 2005.

[21] Nasa guidebook for safety critical software - analysis and development. NASA Lewis Research Center, NASA-GB-8719.13, 1995. 
[22] Padovan S. Casotto S. Detecting body tides and librations of europa with an altimetric exploration mission. In Astrodynamics Specialist Conference, number AIAA 2008-7200, Honolulu, August 2008.

[23] Agnolon D. et al. Study overview of the near earth asteroid sample return. ESA, SCI-PA/2007/004/PA, 2007.

\section{Appendix A - Notation}

A, body state matrix

$A_{s}$, Asymptotically stable matrix

B, control matrix

Coast $^{-}$, negative turn off thrust switching value, deg

Coast $^{+}$, positive turn off thrust switching value, deg

$\boldsymbol{d}$, disturbance vector

DB, dead band magnitude, deg

Fire $^{-}$, negative turn on thrust switching value, deg

Fire $^{+}$, positive turn on thrust switching value, deg

$F_{\text {Drill }}$, drill reaction force, $\mathrm{N}$

$F x_{\text {base }}$, lateral force acting on base, $\mathrm{N}$

$F x_{\text {leg }}^{L}$, lateral force acting on left leg, $\mathrm{N}$

$F x_{l e g}^{R}$, lateral force acting on right leg, $\mathrm{N}$

$F z_{\text {base }}$, vertical force acting on base, $\mathrm{N}$

$F z_{\text {leg }}^{\text {free }}$, vertical force acting on free to translate leg, $\mathrm{N}$

$F z_{\text {leg }}^{L}$, vertical force acting on left leg, N

$F z_{\text {leg }}^{R}$, vertical force acting on right leg, $\mathrm{N}$

$\mathrm{g}$, gravitational constant, $\mathrm{m} / \mathrm{sec}^{2}$

$\mathrm{I}$, inertia of lander, $\mathrm{kg}-\mathrm{m}^{2}$

$I_{T}$, total impulse, N-sec

$k_{r}$, ratchet constant, $\mathrm{N} / \mathrm{m}$

$k_{s}$, spring constant, $\mathrm{N} / \mathrm{m}$

$\boldsymbol{K}$, real time control variable vector

$K_{s}$, stability inducing matrix

$L_{B}$, base length, $\mathrm{m}$

$L_{L}$, leg natural length, m

$L_{S}$, leg stroke length, $\mathrm{m}$

$m_{B}$, base mass, $\mathrm{kg}$

$m_{L}$, leg mass, $\mathrm{kg}$

$M$, total moment acting on lander center of gravity, N-m

$M_{T}$, moment acting on lander center of gravity from thrusters, N-m

$\mathrm{N}$, Number of thrusters

$\mathbf{P}$, positive definite matrix solution to Lyapunov equation

$q$, rotational rate of lander, $\mathrm{deg} / \mathrm{sec}$

$R(\mathrm{t})$, radial position of lander, $\mathrm{m}$

$\mathbf{R}$, positive semi-definite controllable matrix 
$t$, time, sec

$\mathrm{T}$, thrust, $\mathrm{kg}$

$T_{\text {lat }}$, lateral thrust component, $\mathrm{N}$

$\boldsymbol{u}$, control vector

$u_{\text {leg }}^{L}$, lateral velocity of lander left leg, $\mathrm{m} / \mathrm{sec}$

$u_{l e g}^{R}$, lateral velocity of lander right leg, $\mathrm{m} / \mathrm{sec}$

$u_{R}$, unit radial vector

$u_{\theta}$, unit tangential vector

$u_{\text {base }}$, lateral velocity of lander base, $\mathrm{m} / \mathrm{sec}$

$V$, total potential of lander physical state

$V_{B O D Y R A T E}$, potential due to body rate

$V_{E U L E R}$, potential due to Euler angles

$w_{\text {base }}$, vertical velocity of lander base, $\mathrm{m} / \mathrm{sec}$

$w_{\text {leg }}^{L}$, vertical velocity of lander left leg, $\mathrm{m} / \mathrm{sec}$

$w_{l e g}^{R}$, vertical velocity of lander right leg, $\mathrm{m} / \mathrm{sec}$

$\boldsymbol{x}$, body state vector

$x_{\text {base }}$, lateral position of lander base, $\mathrm{m}$

$x_{\text {leg }}^{\text {free }}$, lateral position of free to translate leg, $\mathrm{m}$

$x_{\text {leg }}^{L}$, lateral position of lander left leg, $\mathrm{m}$

$x_{\text {leg }}^{R}$, lateral position of lander right leg, $\mathrm{m}$

$\boldsymbol{y}$, system output matrix

$z_{\text {base }}$, vertical position of lander base, $\mathrm{m}$

$z_{\text {base }}^{\text {rest }}$, vertical reference position of lander base at rest, $\mathrm{m}$

$z_{\text {error }}$, vertical position error, $\mathrm{m}$

$z_{\text {leg }}^{\text {free }}$, vertical position of free to translate leg, $\mathrm{m}$

$z_{l e g}^{L}$, vertical position of lander left leg, $\mathrm{m}$

$z_{\text {leg }}^{R}$, vertical position of lander right leg, $\mathrm{m}$

$z_{\text {leg }}^{\text {FreeRest }}$, vertical reference position of free to translate leg, $\mathrm{m}$

$z_{\text {leg }}^{\text {Lrest }}$, vertical reference position of lander left leg at rest, $\mathrm{m}$

$z_{\text {leg }}^{\text {Rrest }}$, vertical reference position of lander right leg at rest, $\mathrm{m}$

$\Gamma$, positive definite shaping matrix

$\theta$, lander attitude, deg

$\theta_{i}$, lander initial incidence, deg

$\theta_{\text {Desired }}$, required lander attitude, deg

$\lambda$, shaping parameter

$\Lambda$, positive definite shaping matrix

$\ddot{\theta}^{\text {Max }}$, acceleration produced from one thruster, $\mathrm{deg} / \mathrm{sec}^{2}$

$\phi$, real time control parameter vector

$\phi$, initial rotation position, deg

$\omega$, body rate vector, $\mathrm{deg} / \mathrm{sec}$ 